\title{
Global stability of separated flows: subsonic flow past corners
}

\author{
Global stability of separated flows
}

Received: 27 April 2009 / Accepted: 12 January 2010

(C) Springer-Verlag 2010

\begin{abstract}
The triple-deck equations for the steady subsonic flow past a convex corner are solved numerically using a novel technique based on Chebychev collocation in the direction normal to the body combined with finite differences in the direction along the flow. The resulting set of nonlinear algebraic equations are solved with Newton linearization and using the GMRES method for the solution of the linear system of equations. The stability of the computed steady flows is then examined using global stability analysis. It is found that for small corner angles, the Tollmien-Schlichting modes are globally unstable and these persist to larger corner angles. Multiple steady state solutions also exist beyond a critical corner angle but these are globally unstable because of the presence of the Tollmien-Schlichting modes.
\end{abstract}

Keywords Separated flow $\cdot$ Global stability $\cdot$ Triple-deck $\cdot$ Subsonic $\cdot$ Tollmien-Schlichting

\section{Introduction}

There are many practical applications such as in the flow past aerofoils where it is important to understand the nature of the boundary layer flow past obstacles and changes in local geometry. In many cases the boundary layer separates in the vicinity of the obstacle and the separated flow is prone to many instability mechanisms. In designing laminar flow aerofoils it is important to understand when such instabilities arise and how they can be controlled.

There are many major difficulties in studying these types of flows. In particular the Reynolds number is very large and using numerical techniques, one needs to be able to resolve the very thin boundary layers and shear layers which are present when working with the full Navier-Stokes equations. Theoretical studies based on using a high Reynolds number approximation are criticized on the grounds that the flow is usually turbulent at the Reynolds numbers when the theory might be said to be applicable. Another obstacle, as first pointed out by [1], is that the mean flow in these applications is truly non-parallel and therefore a local analysis based on the Orr-Sommerfeld equation is inadequate. A stability analysis of such a flow requires either a full numerical approach or a self-consistent analytically based approach. In [2] it is suggested that a high-frequency analysis could overcome some of these difficulties.

In the current work, we have chosen to investigate the separated subsonic flow past convex corners governed by the triple-deck equations. Asymptotic analysis of the Navier-Stokes equations in the limit that the

Communicated by T. Colonius

R. P. Logue · J. S. B. Gajjar $(\bowtie)$

School of Mathematics, University of Manchester, Manchester M13 9PL, UK

E-mail: j.gajjar@manchester.ac.uk

\section{A. I. Ruban}

Department of Mathematics, Imperial College, London SW7 2AZ, UK

E-mail: a.ruban@imperial.ac.uk 
Reynolds number is large is described by triple-deck theory for such flows, see [3,4]. This approach avoids one of the difficulties mentioned above in that with a proper choice of scales, instead of working with the full Navier-Stokes equations, one has a reduced set of equations but which nevertheless can capture some of the essential physics, at least in the thin boundary layer and shear layer regions, see also [2].

The main objectives of the current work are to solve the steady triple-deck equations and compute the separated flows for a range of corner angles, and then analyze the stability of the computed flows. Since the mean flow is non-parallel the stability analysis is based on searching for global modes with perturbations proportional to $e^{-\lambda t}$ and solving a two-dimensional partial differential eigenvalue problem.

In Sect. 2, we discuss the problem formulation and give a brief description of the numerical techniques used. This is followed by a discussion of the main results in Sect. 3, and our conclusions are given in Sect. 4.

\section{Governing equations and numerical techniques}

Consider the flow of a compressible fluid flowing past a corner on the body contour, which locally may be thought of as being made of two flat plates inclined at an angle $\alpha^{*}$, see Fig. 1. We take Cartesian coordinates $\left(x^{*}, y^{*}\right)$ (dimensional quantities will be denoted by an asterisk) with the oncoming flow aligned with the $x^{*}$-axis. The oncoming fluid velocity far upstream is $U_{\infty}^{*}$ in the $x^{*}$-direction, with free-stream pressure $p_{\infty}^{*}$. The Reynolds number $\operatorname{Re}=U_{\infty}^{*} \rho_{\infty}^{*} L^{*} / \mu_{\infty}^{*}$ is large with $L^{*}$ being a characteristic length scale and $\rho_{\infty}^{*}$ the free-stream density, $\mu_{\infty}^{*}$ the free-stream viscosity. We will assume that the corner angle is small of order $O\left(R e^{-1 / 4}\right)$.

In the vicinity of the corner the interaction between the boundary layer and the external flow is described by triple-deck theory, and in the lower-deck of thickness $O\left(R e^{-5 / 8}\right)$ and lateral extent $O\left(R e^{-3 / 8}\right)$, the solution properties may be represented as, see [3,4],

$$
\begin{gathered}
t^{*}=R e^{-\frac{1}{4}} \frac{L^{*}}{U_{\infty}^{*}} C_{\infty}^{\frac{1}{4}} \tau^{-\frac{3}{2}} \beta^{-\frac{1}{4}} T_{w}^{\prime} t, \quad x^{*}=L^{*} R e^{-\frac{3}{8}} \tau^{-\frac{5}{4}} C_{\infty}^{\frac{3}{8}} \beta^{-\frac{3}{8}}\left(T_{w}^{\prime}\right)^{\frac{3}{2}} x, y^{*}=L^{*} R e^{-\frac{5}{8}} C_{\infty}^{\frac{5}{8}} \tau^{-\frac{3}{4}} \beta^{-\frac{1}{8}}\left(T_{w}^{\prime}\right)^{\frac{3}{2}} y, \\
u^{*}=U_{\infty}^{*} R e^{-\frac{1}{8}} \tau^{1 / 4} C_{\infty}^{\frac{1}{8}} \beta^{-\frac{1}{8}}\left(T_{w}^{\prime}\right)^{\frac{1}{2}} u, \quad v^{*}=U_{\infty}^{*} R e^{-\frac{3}{8}} \tau^{\frac{3}{4}} C_{\infty}^{\frac{3}{8}} \beta^{\frac{1}{8}}\left(T_{W}^{\prime}\right)^{\frac{1}{2}} v, \\
p^{*}=p_{\infty}^{*}+\rho_{\infty}^{*} U_{\infty}^{* 2} \operatorname{Re}^{-\frac{1}{4}} C_{\infty}^{\frac{1}{4}} \tau^{\frac{1}{2}} \beta^{-\frac{1}{4}} p, \quad \alpha^{*}=\alpha \operatorname{Re}^{-\frac{1}{4}} \tau^{\frac{1}{2}} \beta^{\frac{1}{4}}
\end{gathered}
$$

In the above $t^{*}$ is the time, and we have taken the Chapman viscosity law with $C_{\infty}$ being the Chapman constant given by $\left(C_{\infty}=\mu_{w}^{*} T_{\infty}^{*} / \mu_{\infty}^{*} T_{w}^{*}\right)$, and $T_{w}^{\prime}=T_{w}^{*} / T_{\infty}^{*}$ is the ratio of the wall temperature to free-stream temperature. Also $\beta=\left|1-M_{\infty}^{2}\right|$ where $M_{\infty}(<1)$ is the free-stream Mach number and $\tau=\partial U_{\mathrm{B}} / \partial y$ is the value of the wall shear of the oncoming boundary layer flow immediately upstream of the interaction region.

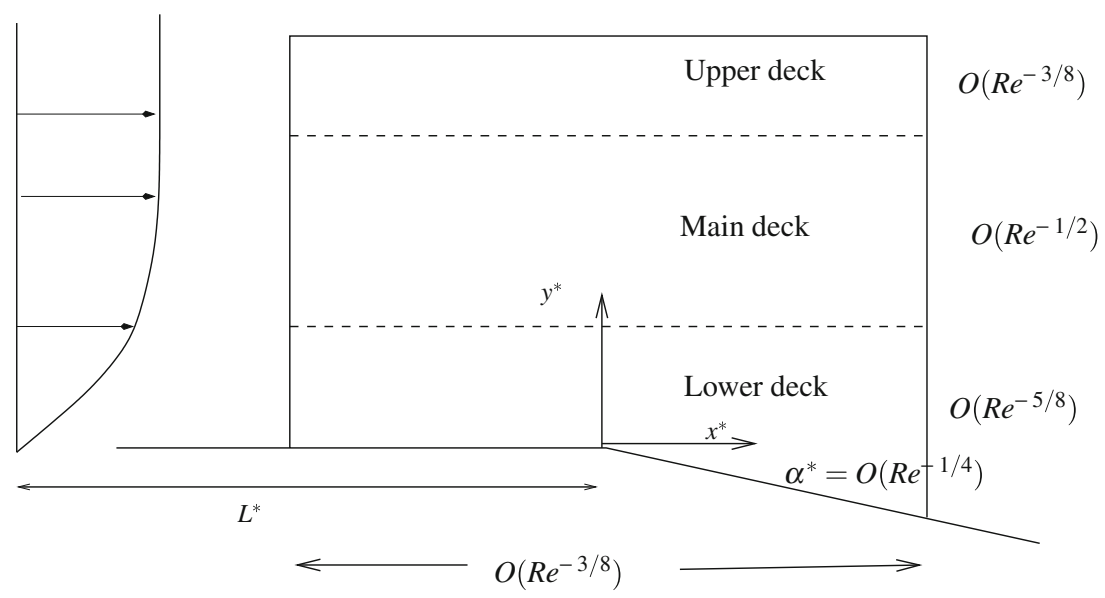

Fig. 1 Schematic of the triple-deck region near the corner 
Instead of a sharp corner it is more convenient to work with the wall shape described by the equation $y=f(x)$ where

$$
f(x)=\frac{1}{2} \alpha\left(x+\sqrt{x^{2}+r^{2}}\right),
$$

with $r=0.1$, and $\alpha$ being the scaled angle parameter.

After substitution of the above into the Navier-Stokes equations and setting $R e \rightarrow \infty$ leads to the unsteady triple-deck equations:

$$
\begin{gathered}
\frac{\partial u}{\partial x}+\frac{\partial v}{\partial y}=0, \\
\frac{\partial u}{\partial t}+u \frac{\partial u}{\partial x}+v \frac{\partial u}{\partial y}=-\frac{\partial p}{\partial x}+\frac{\partial^{2} u}{\partial y^{2}}, \\
p(x, y, t)=P(x, t)=-\frac{1}{\pi} \int_{-\infty}^{\infty} \frac{A^{\prime}(s, t)}{s-x} \mathrm{~d} s .
\end{gathered}
$$

These need to be solved with the boundary conditions

$$
\begin{aligned}
& u=v=0 \text { at } y=f(x), \\
& u \rightarrow y+A(x, t)+\cdots \text { as } y \rightarrow \infty, \\
& u \rightarrow y \text { as } x \rightarrow-\infty \\
& P(x, t) \rightarrow-\frac{\alpha}{\pi} \log |x| \text { as }|x| \rightarrow \infty .
\end{aligned}
$$

Here $(u, v)$ represent the scaled streamwise and normal velocity components, $P$ is the pressure, and $A$ is a scaled displacement function. The only parameter left in the problem is the scaled angle parameter $\alpha$ appearing in the reduced wall shape $f(x)$.

\subsection{Global stability analysis}

The solution of the steady version of the boundary-value problem (2) gives the basic flow state

$$
(u, v)=\left(U_{\mathrm{B}}(x, y), V_{\mathrm{B}}(x, y)\right), \quad P=P_{\mathrm{B}}(x), \quad A=A_{\mathrm{B}}(x)
$$

for a given value of the parameter $\alpha$. In order to perform the stability analysis we look for global modes of the form

$$
\begin{aligned}
(u(x, y, t), v(x, y, t)) & =\left(U_{\mathrm{B}}(x, y), V_{\mathrm{B}}(x, y)\right)+\delta(\tilde{u}(x, y), \tilde{v}(x, y)) e^{-\lambda t}, \\
P(x, t) & =P_{\mathrm{B}}(x)+\delta \tilde{P}(x) e^{-\lambda t}, \quad A(x, t)=A_{\mathrm{B}}(x)+\delta \tilde{A}(x) e^{-\lambda t},
\end{aligned}
$$

where $\delta$ is taken to be small. After substituting into $(2,3)$ and linearizing the equations for small $\delta$ gives rise to a set of linearized stability equations for $(\tilde{u}, \tilde{v}, \tilde{P}, \tilde{A})$ given by

$$
\begin{gathered}
\frac{\partial \tilde{u}}{\partial x}+\frac{\partial \tilde{v}}{\partial x}=0, \quad \tilde{P}=-\frac{1}{\pi} \int_{-\infty}^{\infty} \frac{\tilde{A}^{\prime}(s)}{s-x} \mathrm{~d} s, \\
-\lambda \tilde{u}+U_{\mathrm{B}} \frac{\partial \tilde{u}}{\partial x}+\tilde{u} \frac{\partial U_{\mathrm{B}}}{\partial x}+V_{\mathrm{B}} \frac{\partial \tilde{u}}{\partial y}+\tilde{v} \frac{\partial U_{\mathrm{B}}}{\partial y}=-\frac{\partial \tilde{P}}{\partial x}+\frac{\partial^{2} \tilde{u}}{\partial y^{2}} . \\
\tilde{u} \rightarrow \tilde{A} \text { as } y \rightarrow \infty,
\end{gathered}
$$

and homogeneous boundary conditions. The system of Eqs. 4 constitutes a two-dimensional partial differential eigenvalue problem and non-trivial solutions exist only for certain eigenvalues $\lambda$. 


\subsection{Numerical methods}

The steady equations were solved using a hybrid discretization technique in which we used second-order finite differences in the $x$-direction and Chebychev collocation in the $y$-direction, as for example described in $[5,6]$. The Hilbert integral for the pressure was handled using the same techniques as described in [7]. The nonlinear discrete equations are solved using Newton iteration. One consequence of the discretization of the Hilbert integral, however, is that the linear systems of equations generated no longer have the block-pentadiagonal form as in [5]. Thus, the direct solver as used in [5,6] was replaced by an iterative method using GMRES [8], but with the block pentadiagonal solver as the preconditioner.

For the eigenvalue problem (4) the same discretization techniques were used. The resulting discrete problem then takes the form

$$
\mathbf{J U}=\lambda \mathbf{B U},
$$

where $\mathbf{U}$ denotes the vector of unknowns and the coefficient matrix $\mathbf{J}$ is the Jacobian matrix of the linearized steady operator, with entries dependent on the basic flow. The matrix $\mathbf{B}$ arises from the unsteady part of the operator and $\mathbf{B}$ is a singular diagonal matrix with the non-zero entries arising from (4b). The generalized eigenvalue problem (5) was solved using ARPACK [9].

Further details of the numerical techniques used may be found in [10].

\section{Results and discussion}

The numerical techniques described above are novel for the solution of the steady triple-deck equations and thus were extensively tested and the results obtained compared with those available from other studies. In general the agreement with previous results, such as [11-13] is excellent for small corner angles, but there exist some minor differences for larger angles, which may be attributed to various factors such as the difference in numerical techniques used and also the grid sizes used here are much finer compared to those used in previous studies.

In the discussion of the results we focus attention on the case of convex corners with $\alpha$ negative as this case has some additional features not found in the concave corner case.

\subsection{Basic flow results, convex corner}

Results for the basic flow for varying corner angles from $\alpha=-1$ to -5 are shown in Fig. 2. Ahead of the corner the flow is strongly attached but the skin friction drops rapidly after the corner. The pressure also drops rapidly ahead of the corner but recovers downstream. The drop in pressure is more pronounced for decreasing corner angle. Figure 2 would suggest that beyond a critical angle, the skin friction becomes negative and there is incipient separation. However, Korolev [14] was the first to notice that the subsonic triple-deck flow past a convex corner has multiple solutions. The solutions shown in Fig. 2 are those on a branch with either no separation or a short separation bubble. In Fig. 3 we have shown a plot of the bubble separation length (defined by the region for which $\left.\tau_{\mathrm{B}}(x)=\partial U_{\mathrm{B}} / \partial y(y=0)<0\right)$ versus angle $\alpha$ and it is clear that there is a second solution branch with a long separation bubble. Our results indicate that there is a turning point bifurcation at $\alpha=-5.58$, with separation on the lower branch occurring at $\alpha=-5.07$ whilst in [7] the corresponding critical values are $\alpha=-5.9$ for the turning point and $\alpha=-5.2$ for separation. We also find (in agreement with [7]) only one turning point bifurcation in the range of angles shown in Fig. 3 in contrast to [14] where multiple bifurcation points are shown, although [14] used a sharp corner in his computations. Figure 3 suggests that another turning point bifurcation may also exist but because of the large gradients and extended separated flow region it was not possible to extend our computations beyond those shown.

In Figs. 4 and 5 we compare the displacement function, pressure and skin friction for the multiple solutions of the two branches for $\alpha=-5.5$ and $\alpha=-4.5$. For the former there is a short separation bubble for the solution on the lower branch and a larger separated bubble on the upper-branch. For the $\alpha=-4.5$ case only the upper-branch solution has a separated flow. For the longer separated bubbles the pressure and skin friction plots show a plateau effect in the reversed flow region, before an abrupt recovery closer to reattachment. 

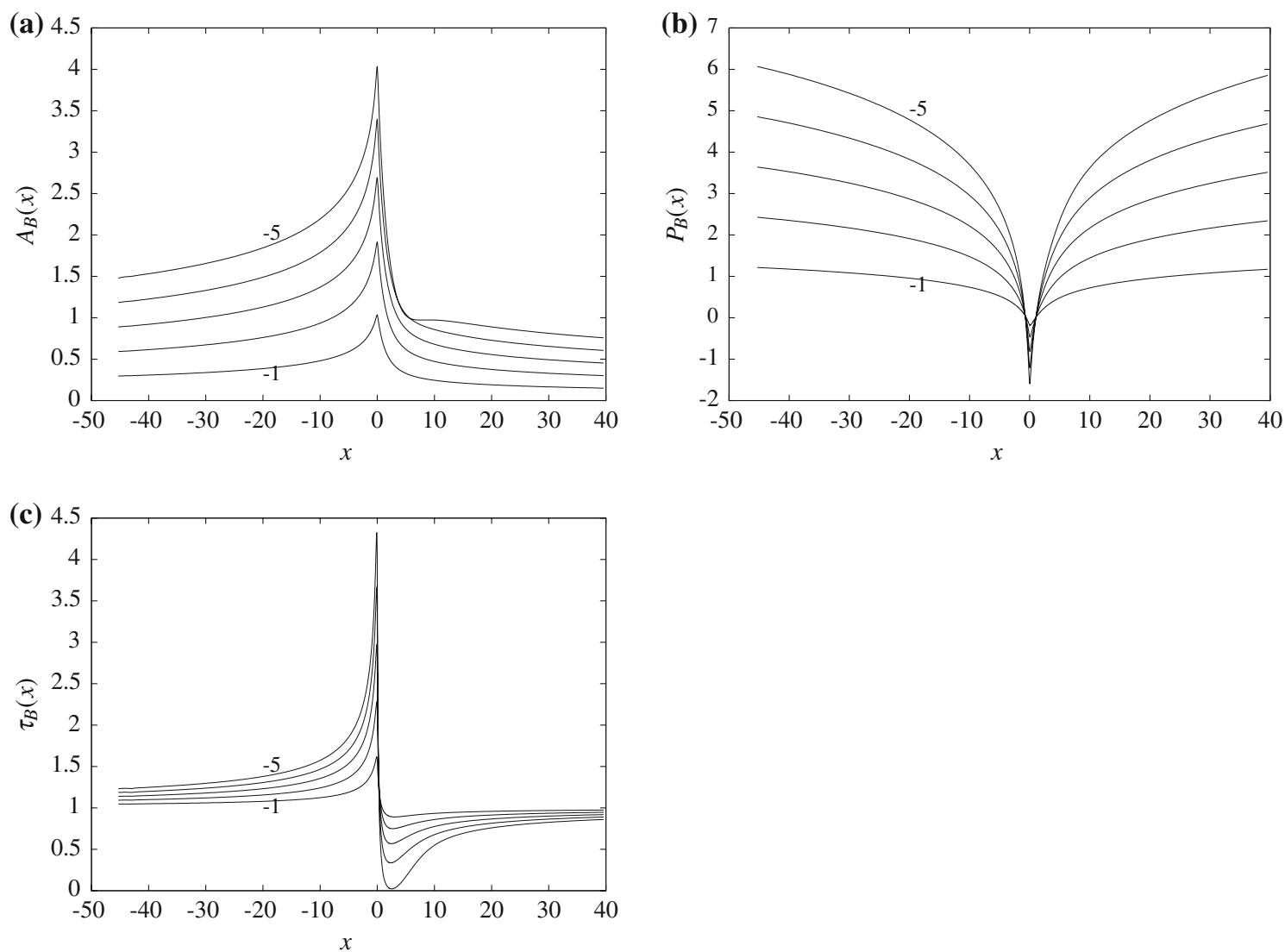

Fig. 2 Plots of the a displacement function $A_{\mathrm{B}}(x)$, b pressure $P_{\mathrm{B}}(x)$ and $\mathbf{c}$ skin friction $\tau_{\mathrm{B}}(x)=\partial U_{\mathrm{B}} / \partial y(y=0)$ for varying corner angles from $\alpha=-1$ to $\alpha=-5$ in steps of -1

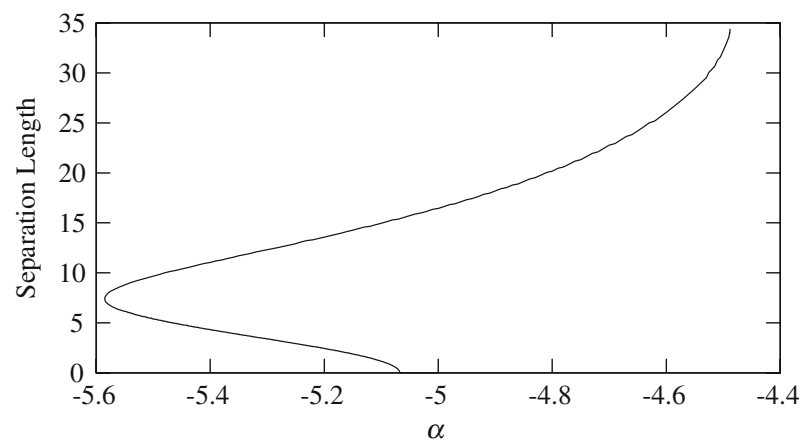

Fig. 3 A plot of the separation length of the bubble versus corner angle

\subsection{Global stability results}

The linear stability of the basic flow was examined using the method based on global stability analysis. It is known that for the flat plate case with $\alpha=0$ the subsonic triple-deck flow is linearly unstable to Tollmien-Schlichting (TS) disturbances of the form $e^{i(k x-\omega t)}$, see [1,2]. In fact for $\alpha=0$ the basic flow given by $U_{\mathrm{B}}=y, V_{\mathrm{B}}=0, P_{\mathrm{B}}=0, A_{\mathrm{B}}=0$ satisfies the Eqs. 2 exactly and therefore it is possible to investigate linear stability properties using a normal mode decomposition in both time and space of the form $e^{i(k x-\omega t)}$. For non-zero $\alpha$ the basic flow is no longer parallel and such a normal mode decomposition cannot be made.

In Fig. 6 we have shown the real and imaginary parts of the eigenvalue spectrum as calculated from the global stability analysis for the flat plate case $\alpha=0$. Clearly the flat plate flow is globally unstable. In computing the eigenfunctions, it was noticed that the perturbations had a growing wave like form spatially, and for 
(a)

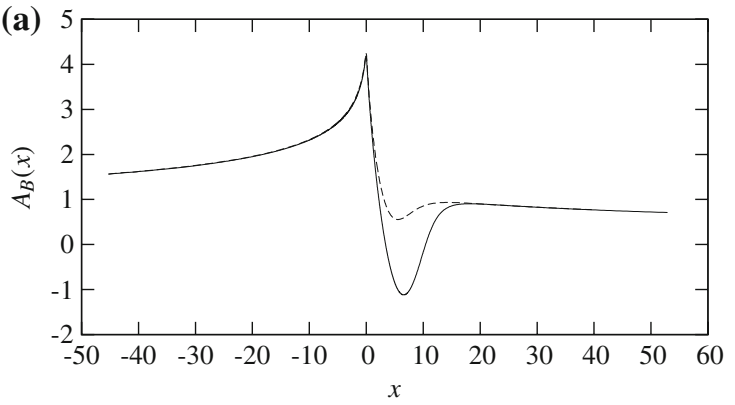

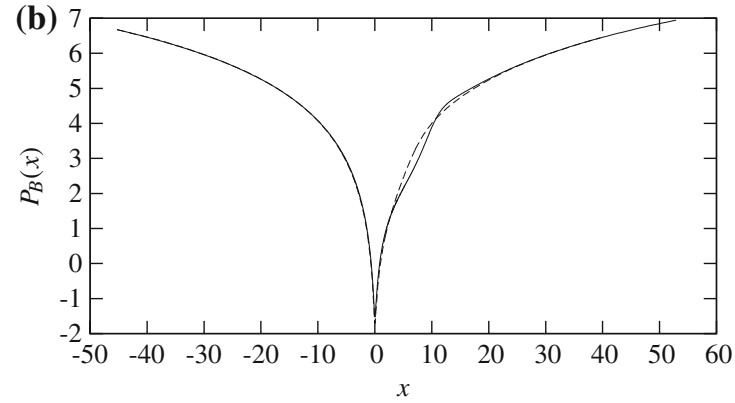

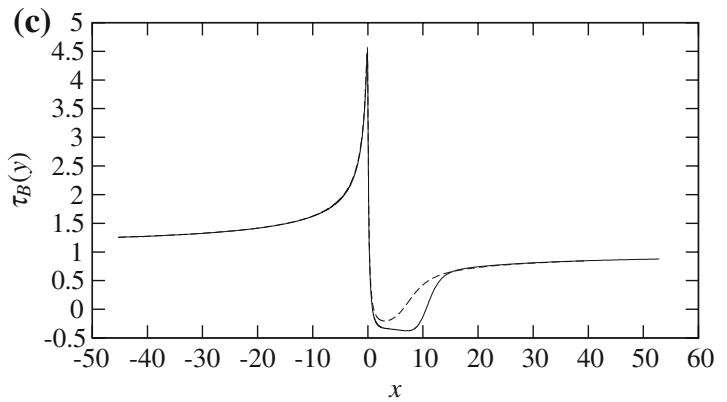

Fig. 4 Plots of the a displacement function $A_{\mathrm{B}}(x)$, b pressure $P_{\mathrm{B}}(x)$ and $\mathbf{c}$ skin friction $\tau_{\mathrm{B}}(x)=\partial U_{\mathrm{B}} / \partial y(y=0)$ for $\alpha=-5.5$. The dashed line indicates the solution with the shorter separated bubble
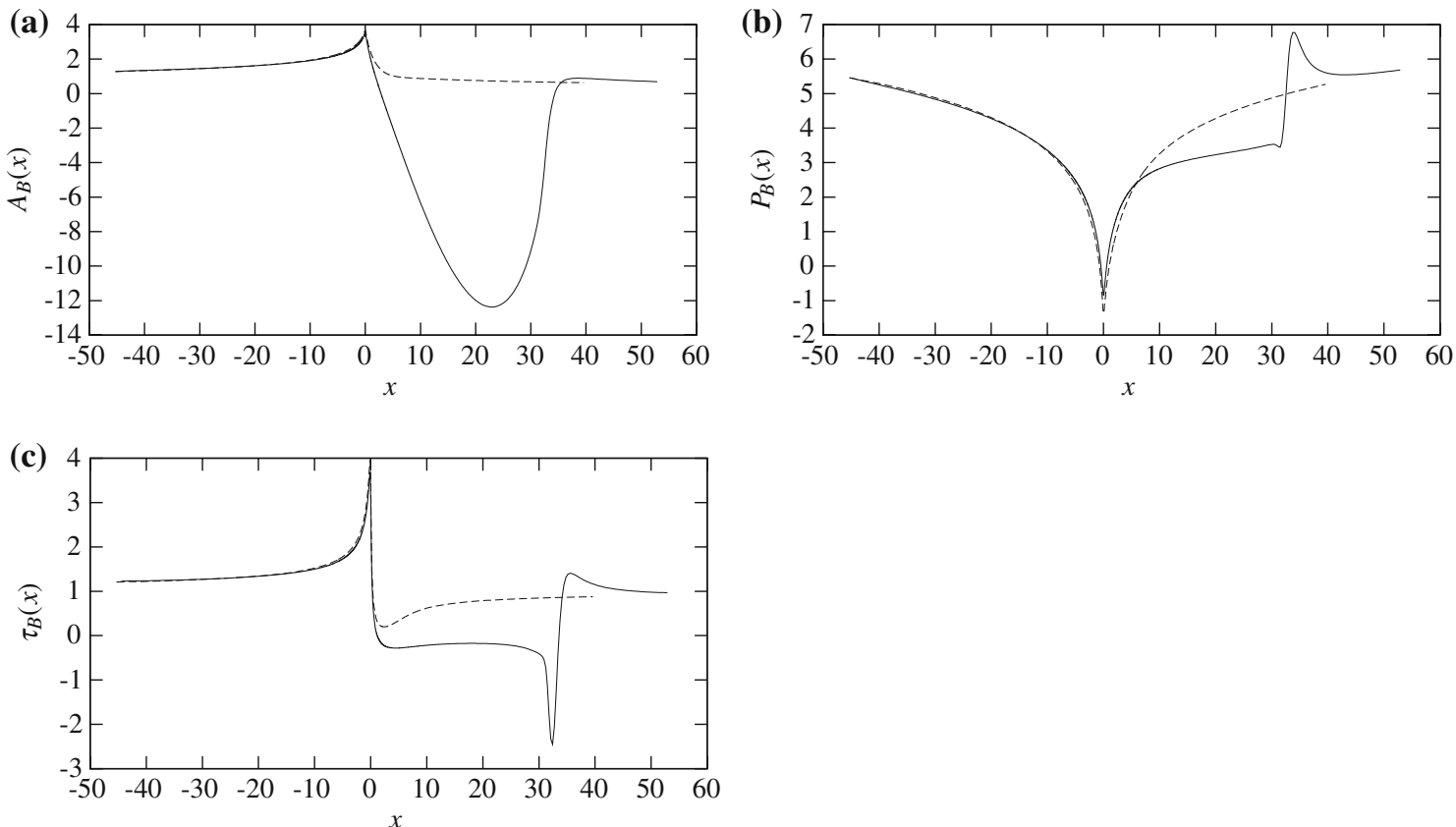

Fig. 5 Plots of the a displacement function $A_{\mathrm{B}}(x)$, b pressure $P_{\mathrm{B}}(x)$ and $\mathbf{c}$ skin friction $\tau_{\mathrm{B}}(x)=\partial U_{\mathrm{B}} / \partial y(y=0)$ for $\alpha=-4.5$. The dashed line indicates the solution on the lower branch with no separation

this reason the grid size convergence properties were improved by modifying the global stability operator to have a downstream condition of the form

$$
\frac{\mathrm{d}^{2} \tilde{p}}{\mathrm{~d} x^{2}}=-\gamma^{2} \tilde{p}
$$



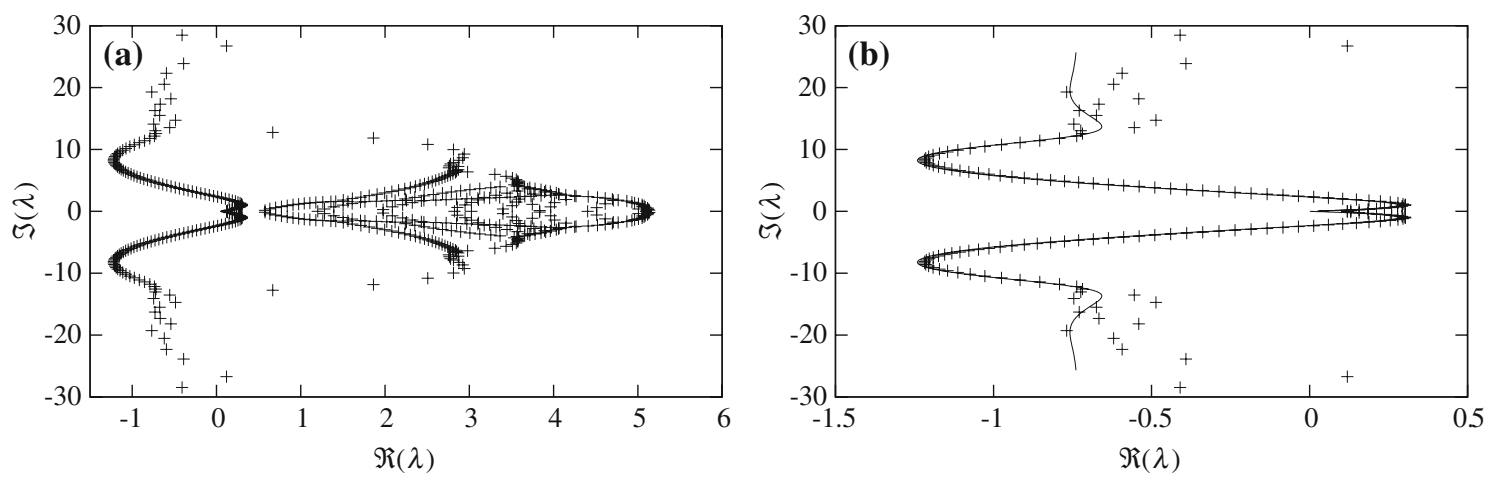

Fig. 6 a The eigenvalue spectrum for the flat plate with $\alpha=0$ with $N=50$ and $M=5001$. b A comparison of the results from the global stability calculations (symbols) for $\alpha=0$ with the analytic results (solid line) from Eq. (7)
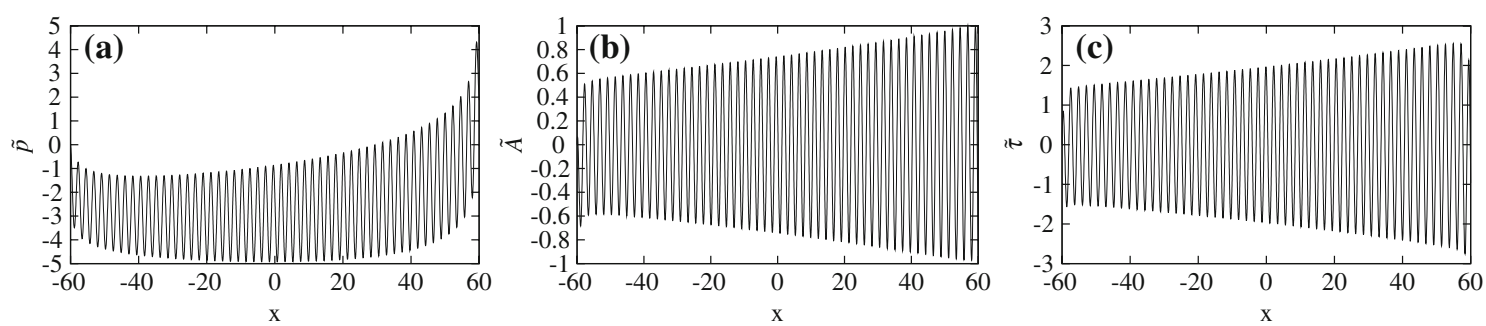

Fig. 7 a Eigenfunctions for the flat plate with $\alpha=0$ showing a the pressure $\tilde{p}$, $\mathbf{b}$ the displacement $\tilde{A}$, and the perturbation skin friction $\tilde{\tau}=\frac{\partial \tilde{u}}{\partial y}(x, y=0)$

for the pressure. Here the factor $\gamma$ was unknown at the outset but was computed iteratively. Similar conditions have been used by [15] for linear stability investigations for the flow over a flat plate.

In Fig. 6a the most unstable eigenvalue is given by $\lambda=-1.21805 \pm i 8.35077$ using a grid of $N=$ $80, M=5001$ points. The eigenfunctions corresponding to the most unstable mode are shown in Fig. 7 . The plots suggest that the unstable wave is not only growing in time but also spatially as well.

For the flat plate it is possible to calculate the dispersion relations for perturbations proportional to $e^{i k x-\lambda t}$, and assuming that $k$ is real one obtains the dispersion relation,

$$
(i k)^{\frac{1}{3}} \frac{k^{2}}{|k|}=\frac{\operatorname{Ai}^{\prime}\left(\xi_{0}\right)}{\int_{\xi_{0}}^{\infty} \operatorname{Ai}(\xi) \mathrm{d} \xi}, \quad \xi_{0}=-(i k)^{-2 / 3} \lambda
$$

where we take a branch cut such that $-3 \pi / 2<\arg (k)<\pi / 2$. The properties of (7) are discussed in many places, see for example [16,17]. For a given $k$ there is a continuous spectrum of modes $\lambda=\lambda(k)$ as can be seen also from the properties of (7) for small $k$ when $\lambda \sim-(i k)^{2 / 3} a_{n}^{\prime}$ where $a_{n}^{\prime}$ is a zero of the derivative of the Airy function $\mathrm{Ai}^{\prime}(\mathrm{z})$. A numerical solution of (7) for the most unstable branch of eigenvalues is compared with the results of the global stability analysis in Fig. $6 \mathrm{~b}$ and this demonstrates that the unstable global modes are TS modes.

Of interest is the change in the spectrum with increasing corner angle $\alpha$ and Fig. 8 shows the spectrum for different values of $\alpha$. We can see that the TS modes persist for non-zero values of $\alpha$. It is interesting that in the vicinity of the origin, the spectrum remains almost independent of $\alpha$ which suggests that the dispersion relation (7) may be used to describe the behaviour of the eigenvalues for non-zero $\alpha$. Figure 8 shows that the most unstable flat plate mode is stabilized for increasing $\alpha$ but the stability computations for non-zero $\alpha$ also picked up more unstable modes with large imaginary parts of $\lambda$, as seen in Fig. 8 for $\alpha=2$ and $\alpha=3$. The modes with large imaginary parts are also present for $\alpha=0$ but are only seen when a sufficiently fine grid in the $x$-direction is used. The presence of these modes would suggest that time simulations with the linearized unsteady equations for both zero and non-zero $\alpha$, would be severely affected by high frequency oscillatory motion growing fast with increasing time.

Consider next the stability of the multiple solutions discussed earlier. Taken in isolation, the bifurcation diagram Fig. 3 with a single turning point would suggest that one branch of solutions is stable and the other 


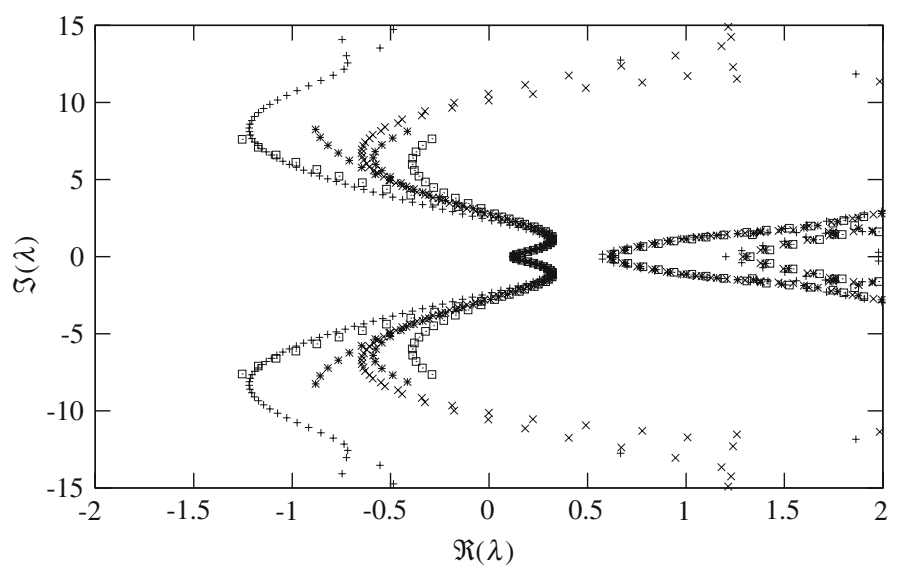

Fig. 8 Spectrum for different values of $\alpha$ with the plus symbols for $\alpha=0$, times for $\alpha=1$, asterisks for $\alpha=2$ and squares for $\alpha=3$

Table 1 Converged eigenvalues for the short bubble solution and long bubble solutions

\begin{tabular}{llll}
\hline Angle & Eigenvalue & Angle & Eigenvalue \\
\hline Short bubble solution & & & -0.076 \\
$\alpha=-5.5$ & -0.047 & $\alpha=-5.0$ & -0.075 \\
$\alpha=-5.4$ & -0.062 & $\alpha=-4.9$ & -0.073 \\
$\alpha=-5.3$ & -0.070 & $\alpha=-4.7$ & -0.073 \\
$\alpha=-5.2$ & -0.074 & $\alpha=-4.6$ & -0.072 \\
$\alpha=-5.1$ & -0.076 & $\alpha=-4.5$ & 0.949 \\
Long bubble solutions & & $\alpha=-5.0$ & 0.950 \\
$\alpha=-5.5$ & 0.057 & $\alpha=-4.9$ & 0.952 \\
$\alpha=-5.4$ & 0.089 & $\alpha=-4.7$ & 0.953 \\
$\alpha=-5.3$ & 0.946 & $\alpha=-4.6$ & 0.954 \\
$\alpha=-5.2$ & 0.947 & $\alpha=-4.5$ & \\
$\alpha=-5.1$ & 0.948 & & \\
\hline
\end{tabular}

branch is unstable. The presence of the turning point is confirmed by the change in the eigenvalue spectrum in Table 1, where we have shown the values of the real eigenvalue for the two solutions as $\alpha$ changes near the turning point. This eigenvalue moves towards the origin along the real axis as the turning point is approached. However in Fig. 9 the full spectrum for the two solutions shows that even though a consideration of the real eigenvalue changing signs might suggest that the short bubble branch is stable and the long bubble branch unstable, both solutions are unstable because of the presence of the TS modes.

\section{Conclusions}

A new numerical method based on a hybrid discretization scheme and using GMRES iteration with a block pentadiagonal preconditioner, has been developed for the solution of the triple-deck equations for subsonic flow over corners. This has been successfully used to compute large-scale separated flows and multiple solutions have been found. In general the results agree well with previous work although there exist differences in the values of $\alpha$ corresponding to the turning point and the incipience of the separation on the lower solution branch.

The stability of the computed basic flows has been examined by investigating perturbations of the form $e^{-\lambda t} g(x, y)$ and solving a two-dimensional partial differential eigenvalue problem for the eigenfunctions $g$ and eigenvalues $\lambda$. It is found that the globally unstable modes for small corner angle are Tollmien-Schlicting modes and our results are in excellent agreement with analytical results for the flat plate case. For non-zero corner angle, the basic flow is non-parallel and the analytical results no longer hold. It is found that as the corner angle is decreased from zero, the dominant TS modes are stabilized for the lower branch solution. In should be noticed that due to the scalings (1) the growth rate is of order $R e^{1 / 4}$ and frequencies of order $R e^{-1 / 4}$ and also given the short spatial scales, numerical simulations with the full Navier-Stokes equations are unlikely to be able to pick up these globally unstable modes unless very fine grids in time and space are used. 


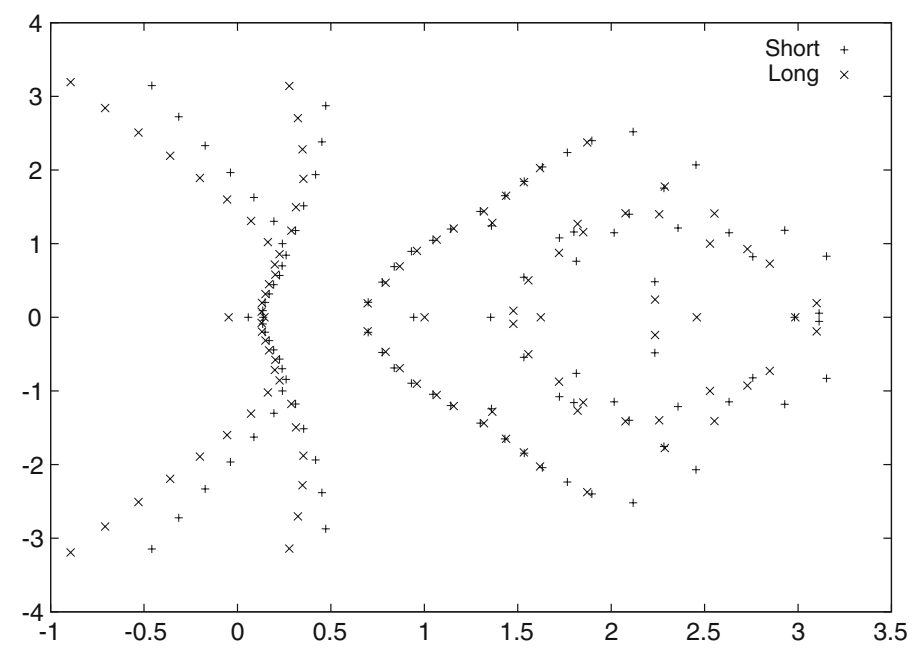

Fig. 9 Spectrum for the short and long bubble solutions when $\alpha=-5.5$, with the short bubble spectrum shown by plus symbols and the long bubble spectrum by the times

The turning point bifurcation is also confirmed via the eigenvalue spectrum which shows a real eigenvalue changing sign as we move from one solution branch to another. However, the presence of other more unstable modes shows that both multiple solution branches are unstable, a result which is particularly important in the context of marginal separation theory.

Finally, the presence of modes in the spectrum with large imaginary parts suggests difficulties in performing numerical simulations of the linearized unsteady triple-deck equations with the same discretization techniques. The presence of these modes is likely to lead to small scale highly oscillatory modes growing rapidly in time with the difficulties being compounded with increasing spatial grid refinement. Similar points have been made by [18] concerning the interactive boundary layer equations.

Acknowledgements JSBG and AR are grateful to the EPSRC for partial support via a grant EP/D050847/1, and RL is grateful for an EPSRC DTA studentship which enabled this work to be done.

\section{References}

1. Smith, F.T.: On the non-parallel flow stability of the Blasius boundary layer. Proc. R. Soc. Lond. A366, 91-109 (1979)

2. Smith, F.T.: Two-dimensional disturbance travel, growth and spreading in boundary layers. J. Fluid Mech. 169, 353-377 (1986)

3. Sychev, V.V., Ruban, A.I., Sychev, V.V., Korolev, G.L.: Asymptotic Theory of Separated Flows. Cambridge University Press, Cambridge (1998)

4. Stewartson, K.: On laminar boundary layers near corners. Q. J. Mech. Appl. Math. 23, 137-152 (1970)

5. Korolev, G.L., Gajjar, J.S.B., Ruban, A.I.: Once again on the supersonic flow separation near a corner. J. Fluid Mech. 463, 173-199 (2002)

6. Boppana, V.B.L., Gajjar, J.S.B.: Global instability in a lid-driven cavity. Int. J. Numer. Methods Fluids 62(18), 827-853 (2009)

7. Kravtsova, M.A., Zametaev, V.B., Ruban, A.I.: An effective numerical method for solving viscous-inviscid interaction problems. Phil. Trans. R. Soc. A 363, 1157-1167 (2005)

8. Saad, Y.: Iterative Methods for Sparse Linear Systems. PWS Publishing Company, New York (1996)

9. Lehoucq, R.B., Sorensen, D.C., Yang, C.: ARPACK User's Guide. S.I.A.M., Philadelphia (1998)

10. Logue, R.P.: Stability and bifurcations governed by the triple-deck and related equations. PhD Thesis, University of Manchester (2008)

11. Ruban, A.I.: On the theory of laminar flow separation of a fluid from a corner point on a solid surface. Uch. Zap. TsaGI. 8(1), 6-11 (1976)

12. Smith, F.T., Merkin, J.H.: Triple-deck solutions for subsonic flow past humps, steps, concave or convex corners and wedged trailing edges. Comput Fluids 10, 7-25 (1982)

13. Turkyilmazoglu M.: Linear absolute and convective instabilities of Some two and three dimensional flows. PhD Thesis, University of Manchester (1998)

14. Korolev, G.L.: Nonuniqueness of separated flow past nearly flat corners. Fluid Dyn. 27, 442-444 (1992)

15. Fasel, H.: Investigation of the stability of boundary layers by a finite-difference model of the Navier-Stokes equations. J. Fluid Mech. 78, 355-383 (1976) 
16. Terentev, E.D.: The linear problem of a vibrator in a subsonic boundary layer. Prikl. Matem. Mekhan. 45(6), 1049-1055 (1981)

17. Ryzhov, O.S., Terentev, E.D.: On the transition mode characterizing the triggering of a vibrator in the subsonic boundary layer on a plate. Prikl. Matem. Mekham. 50, 974-986 (1986)

18. Cowley, S.J.: Laminar boundary-layer theory: a 20th century paradox? In: Aref H., Phillips J.W. (eds.) Proceedings of ICTAM 2000, pp. 389-411. Kluwer (2000) 